Result(s)* 8 OCs occurred during 1277 women screen years; 2 occult OCs at RRSO (both stage 1a), 6 screen-detected OCs (3 prevalent; stage $2 \mathrm{a}, 3$ aii and $3 \mathrm{c}, 3$ incident; stage $1 \mathrm{a}, 3 \mathrm{~b}$ and $4 \mathrm{~b}) .4$ of $6(67 \%)$ screen-detected OCs were diagnosed at stages $<3$ c. 7 of $8(87.5 \%)$ screen-detected cancers were completely cytoreduced. There were no interval cancers. Modelled sensitivity, specificity, PPV and NPV for OC were $87.5 \%$ (CI, 47.3-99.7), 99.9\%(99.9-100), 75\%(34.9-96.8) and $99.9 \%$ (99.9-100) respectively. Economic modelling indicated that surveillance would be cost-saving within the UK National Health Service.

Conclusion* OC surveillance for women declining RRSO in a 'real-word' setting is feasible and equally effective as in research trials, resulting in successful downstaging with likely clinical benefit and healthcare cost savings. Whilst RRSO remains the recommended management for BRCA-carriers, ROCA-based surveillance is a viable interim option for those who defer such surgery.

\section{PREOPERATIVE FDG PET/CT VS CECT IN ADVANCED OVARIAN CANCER}

${ }^{1} \mathrm{~F}$ Boria*, ${ }^{2} \mathrm{M}$ Gutierrez, ${ }^{1} \mathrm{~N}$ Manzour Sifontes, ${ }^{1} \mathrm{E}$ Chacon, ${ }^{1} \mathrm{D}$ Vazquez, ${ }^{1} \mathrm{~T}$ Castellano, 1J Minguez, ${ }^{3} \mathrm{~N}$ Martin, ${ }^{1} \mathrm{~A}$ Gonzalez-Martin, ${ }^{1} \mathrm{~L}$ Sánchez Lorenzo, ${ }^{1} \mathrm{~J}$ Espinos, ${ }^{1} \mathrm{M}$ Cambeiro, ${ }^{1} \mathrm{JL}$ Alcazar, ${ }^{4} \mathrm{~A}$ Alcazxar, ${ }^{5} \mathrm{~L}$ Sancho Rodriguez, ${ }^{1} \mathrm{~L}$ Chiva. ${ }^{1} \mathrm{Cl}$ linica universidad de navarra, Ginecologic Oncology, Madrid, Spain; ${ }^{2}$ Fundación Jimenez Diaz, Madrid, Spain; ${ }^{3}$ Clinica universidad de navarra, Statistics; ${ }^{4}$ Clinica universidad de navarra, Radiology, Spain; ${ }^{5}$ Clinica universidad de navarra, Nuclear medicine

\subsection{6/ijgc-2021-ESG0.662}

Introduction/Background* Ovarian cancer is the leading cause of death from gynecologic cancer in the developed countries. (1)

Multimodality imaging approach with ultrasound, CT, MRI or $\mathrm{PET} / \mathrm{CT}$ is often needed during the diagnosis and prior to the treatment. In AOC, a lot of studies have been done in order to demonstrate a better accuracy in staging advanced disease. Some studies showed PET/CT to be more accurate than traditional CT and MRI imaging, detecting LN metastases, extraabdominal disease and assessing equivocal findings in conventional imaging. (2-6)

Besides, a greater number of distant metastases will be found when using PET/CT as preoperative staging tool and many patients will be upgraded. (7-9).

The aim of this study was to compare the efficacy between preoperative $\mathrm{PET} / \mathrm{CT}$ and ceCT findings according with the final pathology report in patients that underwent surgery for EOC treatment.

Methodology The study is a retrospective unicentric national observational study reviewing data of patients diagnosed with epithelial ovarian cancer that were operated as part of the treatment between July 2018 and February 2021, both included. PET/CT with ceCT started to be used routinely at hour hospital in 2018 for EOC patients. At our institution, the PET/CT is independently evaluated by two imaging specialists: a nuclear medicine doctor (PET-CT) and an expert radiologist in gyn malignancies who evaluate only the CT images.

Result(s)* 56 patients were included in the final analysis. Sensitivity and specificity for PET/CT and CT were: $85 \%$ and $94.3 \%$ vs $80 \%$ and $94.3 \%$ in the ovarie; $38.1 \%$ and $91.2 \%$ vs $23.8 \%$ and $97.1 \%$ in the rectum; $50 \%$ and $86.7 \%$ vs $30 \%$ and $95.6 \%$ in the pelvic nodes; $33.3 \%$ and $97.1 \%$ vs $33.3 \%$ and $97.1 \%$ in the diaphragm; and $25 \%$ and $97.7 \%$ vs $25 \%$ and $100 \%$ in the small bowel mesentery.

When calculating PCI and comparing it to the surgery PCI, PET/CT showed a better intraclass correlation coefficient (0.856) than CeCT (0.751).

Conclusion* Both techniques showed a poor sensitivity and a very good specificity when comparing findings to surgery in the different anatomical places. However, when stimating PCI before surgery, PET/CT showed a better correlation with surgery than CeCT.

\section{OVA-LEAK: PROGNOSTIC SCORE FOR COLO-RECTAL ANASTOMOTIC LEAKAGE IN PATIENTS UNDERGOING OVARIANCANCER SURGERY}

${ }^{1}$ V Lago*, 'B Segarra Vidal, ${ }^{1} \mathrm{P}$ Padilla Iserte, ${ }^{2} \mathrm{~A}$ Gil-Moreno, ${ }^{3} \mathrm{P}$ Cascales, ${ }^{1} \mathrm{MT}$ Luis Javier, ${ }^{2} J \mathrm{~L}$ Sánchez Iglesias, ${ }^{1} \mathrm{M}$ Gurrea, ${ }^{1} \mathrm{~S}$ Domingo. ${ }^{1}$ University hospital La Fe; ${ }^{2}$ University Hospital Val d'Hebron; ${ }^{3}$ Clinic and University Hospital la Arrixaca

\subsection{6/ijgc-2021-ESG0.663}

Introduction/Background* In advanced ovarian cancer surgery, there is rather limited published evidence, drawn from a small sample, providing information about risk factors for anastomotic leak.

Methodology In our previous work, Twelve pre-/intraoperative variables were analysed as potential independent risk factors for anastomotic leak. A predictive model was created to stablish the risk of anastomotic leak for a given patient.

Result(s)* The validation of our proposed predictive model will emerge from the collaborative research performed by 12 centers: 6 from Spain, 2 from United Kingdom, 1 from Italy, 1 from France and 2 from Germany.

Conclusion* Due to the low incidence of AL in ovarian cancer patients, a restrictive stoma policy based on the presence of risk factors should be the actual recommendation. The AL risk for each patient can be predicted by our multivariate model.

\section{CHARACTERISTICS OF BRAIN METASTASIS IN OVARIAN CANCER PATIENTS}

1;2 T Safra*, 1;2E Shahar, 'L Adar, ${ }^{1 ; 2}$ S Peleg Hassom, ${ }^{1}$ A Harpaz, ${ }^{1 ; 2} \mathrm{D}$ Limon. ${ }^{1} \mathrm{Tel}$ Aviv Medical Center; ${ }^{2}$ Tel Aviv University, Tel Aviv-Yafo, Israel

\subsection{6/ijgc-2021-ESG0.664}

Introduction/Background* Brain metastasis (BM) are uncommon among ovarian cancer (OC) patients. Their frequency, risk factors and clinical repercussions are not well described. We assessed OC patients who developed BM, the role of BRCA status and survival implications.

Methodology We retrospectively evaluated 927 consecutive OC patients treated at our center in 2002-2020.

Result(s)* 28/927(2.9\%) were diagnosed with BM and compared to non-BM cohort. Median age was 60 in both groups, stage III-IV at diagnosis was more common among BM group (96.4\% vs. $84.8 \%, p=0.0065)$ while platinum sensitivity was similar(92.3\% in BM vs. $80.8 \%$ in non-BM, $\mathrm{p}=0.2193) .658$ patients tested for BRCA, 33.6\% $(\mathrm{n}=221)$ were mutation carriers(BRCA+). Of the patients with $\mathrm{BM}, 13 / 22$ tested were carriers. BRCA+ was significantly higher in the BM group $(59.1 \%$ vs. $32.9 \%, p=0.0123)$. The rate of $\mathrm{BM}$ was higher in the BRCA+ compared to BRCA- $\operatorname{group}(5.8 \%$ vs. $2.1 \%$, 